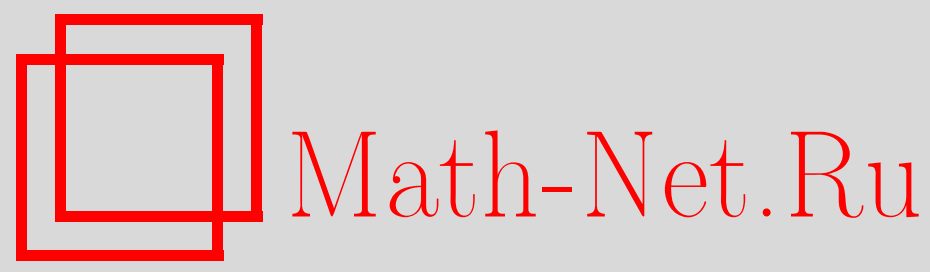

С. Р. Габдрахманов, Б. С. Клебанов, В. В. Филиппов, Об асимптотическом интегрировании квазилинейных дифференциальных уравнений, Матем. заметки, 2001, том 70, выпуск 3, 346-355

DOI: https://doi.org/10.4213/mzm747

Использование Общероссийского математического портала Math-Net.Ru подразумевает, что вы прочитали и согласны с пользовательским соглашением http://www . mathnet.ru/rus/agreement

Параметры загрузки:

IP : 54.162 .27 .143

26 апреля 2023 г., $12: 57: 43$

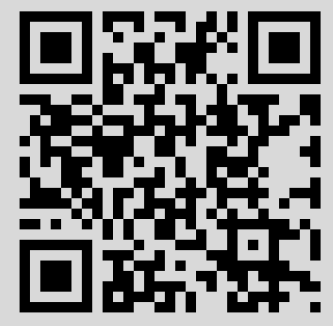


УДК 517.925 .51

\title{
ОБ АСИМПТОТИЧЕСКОМ ИНТЕГРИРОВАНИИ КВАЗИЛИНЕЙНЫХ ДИФФЕРЕНЦИАЛЬНЫХ УРАВНЕНИЙ
}

\author{
С. Р. Габдрахманов, Б. С. Клебанов, В. В. Филиппов
}

\begin{abstract}
С помощью понятия сходимости пространств решений доказаны теоремы об асимптотическом поведении решений систем уравнений, близких к линейньм. Приведен пример использования одной из теорем.

Библиография: 4 названия.
\end{abstract}

1. Введение. Цель статьи - выявить содержание теоремы о существовании решений квазилинейных уравнений с заданной асимптотикой (см. $[1$, гл. Х]) с точки зрения топологических структур, описанных в [2] и [3, гл. IX]. Мы заменяем обычные метрические оценки возмушения типа неравенств и "o-малых" на условия сходимости в соответствующем пространстве $R_{c}(U)$.

Обозначениями и понятиями теории пространств решений дифференциальных уравнений, предложенной В.В. Филипповым, будем пользоваться без дополнительных пояснений (см. [2]). При этом распространим обозначения, относящиеся к открытому подмножеству $M \subseteq \mathbb{R} \times L$, на произвольное $M \subseteq \mathbb{R} \times L$, где $L$ - конечномерное евклидово пространство. Заметим, что если $M \subseteq U \subseteq \mathbb{R} \times L, Z \in R(U)$, то множество $Z_{M}=\{z \in Z: \operatorname{Gr} z \subseteq M\}$ принадлежит $R(M)$.

Пусть $M \subseteq \mathbb{R} \times L$ и $X \in R_{c}(M)$. Через $X^{+}$обозначается пространство максимально продолженных вправо функций из $X$ : функция $z$ принадлежит $X^{+}$, если область определения $z$, обозначаемая $\pi(z)$, либо

1) отрезок $[a, b]$,

2) полуинтервал $[a, b)$,

$3)$ полуинтервал $[a, \infty)$,

причем не существует такой функции $x \in X$, что $\pi(x)=[a, c],\left.x\right|_{\pi(z)}=z$ и $c>b$ в случае 1$), c \geqslant b$ в случае 2 ).

Пусть $S \subseteq M$. Множество $\cup\{\operatorname{Gr} z: z \in X,(\inf \pi(z), z(\inf \pi(z))) \in S\}$ обозначим через $X^{+} S$ (другими словами, $X^{+} S$ - множество всех точек $M$, лежаших на графиках функций из $X^{+}$, исходящих из множества $S$ ).

Лемма 1. Пусть $M \subseteq \mathbb{R} \times \mathbb{R}^{n}, X \in R_{c}(M)$ и $X$ принадлежат все функиии, графики которых одноточечные подмножсества $M$.

Тогда $X$ - компакт в том и только том случае, когда $M$ - компакт.

Работа выполнена при поддержке Российского фонда фундаментальных исследований, грант № 94-01-01695, и Международного научного фонда, грант № J55100. 
ДокАЗАТЕЛЬСтво. Необходимость очевидна. Достаточность следует из условия $X \in R_{c}(M)$ и определения $R_{c}(M)$.

2. Обобщение теоремы Важевского. Здесь рассматривается вариант обобщения топологического принципа Важевского (см. [1]), необходимый для доказательства основного результата.

Пусть множество $U$ открыто в $\mathbb{R} \times L$. Всюду в этом разделе оно считается основным объемлющим пространством, так что для $M \subseteq U$ под [M] мы будем понимать замыкание $M$ в $U$.

Лемма 2. Пусть $S \subseteq M \subseteq U, S-$ компакт, $M$ замкнуто в $U$. Допустим, что $Z \in R_{c e}(U)$.

Тогда существование функиии $z \in Z^{+}$, график которой начинается в $S$ (т.е. $(\inf \pi(z), z(\inf \pi(z))) \in S)$ и лежит в $M$, әквивалентно некомпактности в $C_{s}(U)$ множества $\left\{z \in Z_{M}:(\inf \pi(z), z(\inf \pi(z))) \in S\right\}$.

ДоказАтельство. І. Необходимость. Пусть $z \in Z^{+}, \pi(z)=\left[t_{0}, \omega\right), \omega \in \mathbb{R} \cup\{+\infty\}$, $\left(t_{0}, z\left(t_{0}\right)\right) \in S, \operatorname{Gr} z \subseteq M$. Пусть точки $t_{k}, k \in \mathbb{N}$, лежат в области определения $z$ и стремятся к $\omega$. Тогда из последовательности функций $\left.z\right|_{\left[t_{0}, t_{k}\right]}, k \in \mathbb{N}$, нельзя выбрать сходящуюся подпоследовательность (иначе в $Z$ существует функция, являющаяся продолжением $z$ вправо).

II. Достаточность. Пусть функции $z_{i} \in Z_{M}$ таковы, что $t_{i}=\inf \pi\left(z_{i}\right), y_{i}=z_{i}\left(t_{i}\right)$, $\left(t_{i}, y_{i}\right) \in S$, и из последовательности $z_{i}$ нельзя выбрать сходящейся подпоследовательности. Так как $S$ - компакт, можно считать, переходя, если нужно, к подпоследовательности, что $\left(t_{i}, y_{i}\right) \rightarrow\left(t_{0}, y_{0}\right) \in S$ при $i \rightarrow \infty$.

Пусть $\zeta_{i} \in Z^{-+}-$какое-нибудь продолжение $z_{i}$. Тогда по теореме IX.3.12 из [3] существуют подпоследовательность $\left\{\zeta_{i_{k}}: k \in \mathbb{N}\right\}$ последовательности $\left\{\zeta_{i}: i \in \mathbb{N}\right\}$ и функция $\zeta \in Z^{-+}$такие, что $y_{0}=\zeta\left(t_{0}\right)$ и на любом отрезке из области определения $\zeta$ функции $\zeta_{i_{k}}$ равномерно сходятся к $\zeta$.

Пусть $\zeta_{0}=\left.\zeta\right|_{\left[t_{0},+\infty\right) \cap \pi(\zeta)}$. Очевидно, $\zeta_{0} \in Z^{+}$. Покажем, что $\operatorname{Gr} \zeta_{0}$ лежит в $M$. Предположим противное. Тогда существует такое $\tau \in \pi\left(\zeta_{0}\right)$, что $\left(\tau, \zeta_{0}(\tau)\right) \notin M$. Так как $U \backslash M$ открыто, то, начиная с некоторого $k$, точки $\left(\tau, \zeta_{i_{k}}(\tau)\right)$ также не принадлежат $M$. Так как Gr $z_{i_{k}}$ лежит в $M$, отсюда следует, что $\sup \pi\left(z_{i_{k}}\right)<\tau$. Иначе говоря, функции $z_{i_{k}}$ являются ограничениями функций $\zeta_{i_{k}} \mid\left[t_{i_{k}}, \tau\right]$, сходящихся к $\left.\zeta_{0}\right|_{\left[t_{0}, \tau\right]}$. Но отсюда следует, что и из последовательности $z_{i_{k}}$ можно выбрать сходящуюся подпоследовательность, что противоречит сделанному предположению о функциях $z_{i}$.

Лемма доказана.

СледствИЕ 1. В предположениях леммы 2 существование функиии $z \in Z^{+}$, график которой начинается в $S$ и лежит в $M$, әквивалентно некомпактности мнохества $\left(Z_{M}\right)^{+} S$.

ДоказАтельство. Пусть $\left(Z_{M}\right)^{+} S=A$. Компактность $A$ в силу леммы 1 эквивалентна компактности $Z_{A}$. Заметим теперь, что $Z_{A}$ является множеством таких функций $z$ из $Z_{M}$, что существует продолжающая $z$ функция из $Z_{M}$, график которой начинается в $S$. Таким образом, компактность $Z_{A}$ эквивалентна компактности множества $\left\{z \in Z_{M}:(\inf \pi(z), z(\pi(z))) \in S\right\}$. Отсюда по лемме 2 получаем требуемое. 


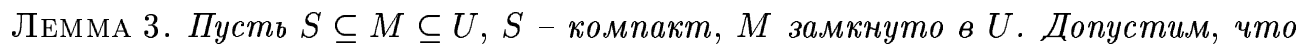
пространства $Z_{i} \in R_{\text {сеи }}(U), i \in \mathbb{N}, u Z \in R_{c e}(U)$ таковь, что последовательность $\left\{Z_{i}: i \in \mathbb{N}\right\}$ сходится $\kappa Z$ в $U$. Пусть, кроме того, множество $\left(Z_{M}\right)^{+} S-$ компакт, а $G$ - его окрестность в $U$.

Тогда, начиная с некоторого $i$, множества $\left(\left(Z_{i}\right)_{M}\right)^{+} S$ также компактны $u$ лежат в $G$.

ДокАЗАтЕльство. І. Докажем вначале компактность. Предположим противное. Тогда согласно следствию 1 существует такая последовательность функций $z_{k} \in Z_{i_{k}}^{+}$ $\left(i_{1}<i_{2}<\cdots\right)$, что $\left(\inf \pi\left(z_{k}\right), z_{k}\left(\inf \pi\left(z_{k}\right)\right)\right) \in S$ и $\operatorname{Gr} z_{k} \subseteq M$. Тогда по теореме IX.3.12 из [3] существует функция $z \in Z^{+}$, обладающая теми же свойствами. Применяя следствие 1 , получаем противоречие с компактностью $\left(Z_{M}\right)^{+} S$.

Тем самым, компактность доказана.

II. Докажем оставшуюся часть утверждения леммы. Допустим, что она не верна. Тогда существует такая последовательность функций $z_{k} \in Z_{i_{k}}\left(i_{1}<i_{2}<\cdots\right)$, что $t_{k}=\inf \pi\left(z_{k}\right), s_{k}=\sup \pi\left(z_{k}\right),\left(t_{k}, z_{k}\left(t_{k}\right)\right) \in S,\left(s_{k}, z_{k}\left(s_{k}\right)\right) \in \partial G$ и Gr $z_{k} \subseteq M \cap[G]$. Отметим, что без потери общности можно считать, что $[G]$ - компакт. Так как $\operatorname{Gr} z_{k}$ лежит в компакте $[G]$, в силу сходимости $\left\{Z_{k}: k \in \mathbb{N}\right\}$ к $Z$ в $U$ существует подпоследовательность $z_{k_{j}}$ последовательности $\left\{z_{k}: k \in \mathbb{N}\right\}$, которая сходится к $z \in Z$, причем $(\inf \pi(z), z(\inf \pi(z))) \in S,(\sup \pi(z), z(\sup \pi(z))) \in \partial G$ и $\operatorname{Gr} z \subseteq M$. Таким образом, Gr $z$ лежит в $\left(Z_{M}\right)+S$, но не лежит в $G$. Это противоречит определению $G$.

Лемма доказана.

Перейдем теперь к основной теореме этого пункта. Рассмотрим следующую ситуацию: $M$-замкнутоеподмножество $U, S \subseteq M, H \subseteq M, \partial M=F_{0} \cup F_{1} ; Z$-пространство, принадлежащее $R_{c e}(U)$. Наша задача состоит в поиске функций из $Z^{+}$, графики которых начинаются в $S$ и не выходят за пределы множества $M$.

В следующей теореме под $F_{0}$ понимается та часть гранищы $M$, через которую графики функций из $\left(Z_{M}\right)^{+}$, начинающиеся в $S$, вьйти не могут: $\left(Z_{M}\right)^{+} S \cap F_{0}=\varnothing$ (в частности, $S \cap F_{0}=\varnothing$, т.е. $\left.S \cap \partial M=S \cap F_{1}\right)$. Через точки множества $F_{1}$ эти графики выйти могут, но при этом вьполнено следующее условие:

(*) если график функции $z \in Z_{M}$ начинается в $S$, то в $\pi(z)$ не существует таких чисел $a \leqslant b \leqslant c$, что $(a, z(a)) \in F_{1},(c, z(c)) \in F_{1},(b, z(b)) \in H$.

В условии $(*)$ множество $H$ характеризует “глубину", на которую графики функций из $Z_{M}$, выходящие на границу $M$, могут возвращаться внутрь $M$.

Tеорема 1. Пусть $S \subseteq M \subseteq U, H \subseteq M, \partial M=F_{0} \cup F_{1}, S$ - компакт, множества $H, M, F_{0}$ замкнуты в $U$. Пусть $Z \in\left[R_{\text {сеи }}(U)\right]$. Допустим также, что $\left(Z_{M}\right)^{+} S \cap F_{0}=\varnothing$ и выполнено условие $(*)$.

Если при этом $S \cap F_{1}(=S \cap \partial M)$ является ретрактом множества $M \backslash$ (Int $H \cup$ $\left.F_{0}\right)$, но не является ретрактом $S$, то существует такая функиия $z \in Z^{+}$, что $(\inf \pi(z), z(\inf \pi(z))) \in S u \operatorname{Gr} z \subseteq M$.

ДоказАтЕльство. І. Докажем вначале теорему в предположении $Z \in R_{c e u}(U)$.

Предположим противное. Тогда в силу следствия $1\left(Z_{M}\right)^{+} S-$ компакт. Пусть $s-$ произвольная точка $S$. Обозначим через $\zeta^{s}$ ту единственную функцию из $Z^{+}$, для которой $s=\left(\inf \pi\left(\zeta^{s}\right), \zeta^{s}\left(\inf \pi\left(\zeta^{s}\right)\right)\right)$. Для каждого $s \in S$ существует число

$$
\beta(s)=\inf \left\{t: t \in \pi\left(\zeta^{s}\right),\left(t, \zeta^{s}(t)\right) \in \partial M\right\} .
$$


Очевидно, $\left.\operatorname{Gr} \zeta^{s}\right|_{(-\infty, \beta(s)] \cap \pi\left(\zeta^{s}\right)} \subseteq M$. Также существует число

$$
\alpha(s)=\inf \left\{t: t \in(-\infty, \beta(s)] \cap \pi\left(\zeta^{s}\right),\left.\operatorname{Gr} \zeta^{s}\right|_{[t, \beta(s)]} \subseteq M \backslash \operatorname{Int} H\right\} .
$$

Покажем, что заданное на $S$ многозначное отображение $F: s \mapsto\left[\inf \pi\left(\zeta^{s}\right), \beta(s)\right]$ полунепрерьвно снизу. Если $s_{k}$ стремится к $s_{0}$ в $S$, то в силу того, что $Z \in R_{c e u}(U)$, функции $\left.\zeta^{s_{k}}\right|_{F\left(s_{k}\right)}$ сходятся к $\left.\zeta^{s_{0}}\right|_{\left[\inf \pi\left(\zeta^{\left.\left.s_{0}\right), T\right]}\right.\right.}$ для некоторого $T \in \mathbb{R}$ (их графики лежат в компакте $\left.\left(Z_{M}\right)^{+} S\right)$. Но точка $\left(T, \zeta^{s_{0}}(T)\right)$ лежит на границе $M$ (как предел последовательности $\left.\left(\beta\left(s_{k}\right), \zeta^{s_{k}}\left(\beta\left(s_{k}\right)\right)\right) \in \partial M\right)$, значит, $\beta\left(s_{0}\right) \leqslant T$ и $\beta\left(s_{k}\right) \rightarrow T \geqslant \beta\left(s_{0}\right)$ и потому отображение $F$ полунепрерьвно снизу.

Покажем теперь, что заданное на $S$ многозначное отображение $G: s \mapsto[\alpha(s), \beta(s)]$ также полунепрерьвно снизу. Убедимся вначале, что не существует последовательности $s_{i} \in S$ такой, что $s_{i} \rightarrow s_{0}, \lim _{i \rightarrow \infty} \alpha\left(s_{i}\right)>\beta\left(s_{0}\right)$. В противном случае функции $\left.\zeta^{s_{i}}\right|_{\left[\inf \pi\left(\zeta^{s_{i}}\right), \beta\left(s_{i}\right)\right]}$ сходятся к $\left.\zeta^{s_{0}}\right|_{\left[\inf \pi\left(\zeta^{s_{0}}\right), T_{1}\right]}$, где $\beta\left(s_{i}\right) \rightarrow T_{1}, \alpha\left(s_{i}\right) \rightarrow T_{0}, T_{1} \geqslant T_{0}>$ $\beta\left(s_{0}\right)$, причем точки $\left(T_{1}, \zeta^{s_{0}}\left(T_{1}\right)\right)$ и $\left(\beta\left(s_{0}\right), \zeta^{s_{0}}\left(\beta\left(s_{0}\right)\right)\right)$ лежат на границе $M$ (т.е. в $F_{1}$ в силу условий теоремы), a $\left(T_{0}, \zeta^{s_{0}}\left(T_{0}\right)\right) \in H$ (так как $H$ замкнуто). Это противоречит условию $(*)$. Полунепрерьвность снизу отображения $G: s \mapsto[\alpha(s), \beta(s)]$ теперь легко проверить. Действительно, пусть $s_{i} \rightarrow s_{0}$ и $\lim _{i \rightarrow \infty} \alpha\left(s_{i}\right)=\alpha, \lim _{i \rightarrow \infty} \beta\left(s_{i}\right)=\beta$. Если $s_{0} \in H$, то $\alpha \leqslant \beta\left(s_{0}\right) \leqslant \beta$ и $\zeta^{s_{0}}(\alpha) \in \partial H$, но так как $\alpha\left(s_{0}\right)$ - ближайший из предшествующих $\beta\left(s_{0}\right)$ моментов времени $t$, когда $\zeta^{s_{0}}(t) \in \partial H$, то $\alpha \leqslant \alpha\left(s_{0}\right)$. Если же $s_{0} \in S \backslash H$, то, как легко видеть, $\alpha=\alpha\left(s_{0}\right)=\inf \pi\left(\zeta^{s_{0}}\right)$. Таким образом, в обоих случаях $\alpha \leqslant \alpha\left(s_{0}\right)$. Так как $F$ полунепрерывно снизу, то $\beta \geqslant \beta\left(s_{0}\right)$ и поэтому $\left[\alpha\left(s_{0}\right), \beta\left(s_{0}\right)\right] \subseteq[\alpha, \beta]$. Полунепрерьвность снизу отображения $G$ доказана.

По первой теореме Майкла о селекции (см. [4]) существует такая непрерывная функция $\gamma: S \rightarrow \mathbb{R}$, что $\gamma(s) \in G(s)$ для всех $s$ из $S$. Нетрудно видеть, что функция $\delta: S \rightarrow$ $M \backslash\left(\right.$ Int $\left.H \cup F_{0}\right)$, задаваемая равенством $\delta(s)=\zeta^{s}(\gamma(s))$, непрерывна. Имеем ретракцию $S$ на $S \cap F_{1}$ как композицию $\delta$ с ретракцией $M \backslash\left(\operatorname{Int} H \cup F_{0}\right)$ на $S \cap F_{1}$ (замечая, что для $s \in S \cap F_{1}$ имеем $\beta(s)=\alpha(s)=\inf \pi\left(\zeta^{s}\right)$, т.е. $\left.\delta(s)=s\right)$.

Противоречие с условием теоремы получено, и, тем самым, теорема в предположении $Z \in R_{\text {сеи }}(U)$ доказана.

II. Докажем теперь нашу теорему без этого дополнительного предположения.

Предположим, что утверждение теоремы неверно. Тогда в силу следствия $1\left(Z_{M}\right)^{+} S$ - компакт. Пусть последовательность пространств $Z_{i} \in R_{\text {сеи }}(U)$ сходится к $Z$ в $U$. Пусть, кроме того, $G$ такая окрестность $\left(Z_{M}\right)^{+} S$, что множество $[G]$ компакт и лежит в $U$. В силу леммы 3 , начиная с некоторого $i_{0}$, множества $\left(\left(Z_{i}\right)_{M}\right)^{+} S$ компакты и лежат в $G$. По следствию 1 утверждение теоремы не вьполнено для пространств $Z_{i}$ при $i \geqslant i_{0}$.

Для получения противоречия достаточно показать, что все условия теоремы можно перенести на пространство $Z_{i_{1}}$ для некоторого $i_{1}$.

Докажем вначале, что существует $j \geqslant i_{0}$ такое, что для всех $i \geqslant j$ вьполнено $\left(\left(Z_{i}\right)_{M}\right)^{+} S \cap F_{0}=\varnothing$. Допустим противное. В этом случае существуют такие функции $z_{k} \in Z_{i_{k}}\left(i_{1}<i_{2}<\cdots\right)$, что $\pi\left(z_{k}\right)=\left[t_{k}, T_{k}\right],\left(t_{k}, z_{k}\left(t_{k}\right)\right) \in S,\left(T_{k}, z_{k}\left(T_{k}\right)\right) \in F_{0}$ и $\operatorname{Gr} z_{k}$ лежит в $M$. Тогда $\operatorname{Gr} z_{k}$ лежит в компакте [G]. Используя сходимость последовательности пространств $Z_{i_{k}}$ к $Z$ в $U$, получаем, что существует такая функция $z \in Z$, что $\pi(z)=\left[t_{0}, T_{0}\right],\left(t_{0}, z\left(t_{0}\right)\right) \in S,\left(T_{0}, z\left(T_{0}\right)\right) \in F_{0}$ (здесь важна замкнутость $F_{0}$ в $\left.U\right)$ и $\operatorname{Gr} z \subseteq M$. Это противоречит условию $\left(Z_{M}\right)^{+} S \cap F_{0}=\varnothing$.

Докажем теперь, что существует $l \geqslant j$ такое, что для пространства $Z_{l}$ вьполнено условие $(*)$. Допустим противное. Тогда для каждого $i \geqslant j$ существуют такие 
функции $z_{i} \in Z_{i}$ и числа $t_{i} \leqslant a_{i} \leqslant b_{i} \leqslant c_{i}$, что $\pi\left(z_{i}\right)=\left[t_{i}, c_{i}\right], \operatorname{Gr} z_{i}$ лежит в $M$, $\left(t_{i}, z_{i}\left(t_{i}\right)\right) \in S,\left(a_{i}, z_{i}\left(a_{i}\right)\right) \in F_{1},\left(c_{i}, z_{i}\left(c_{i}\right)\right) \in F_{1},\left(b_{i}, z_{i}\left(b_{i}\right)\right) \in H$. Множества Gr $z_{i}$ лежат в компакте $[G]$, значит, используя сходимость последовательности пространств $Z_{i}$ к $Z$ в $U$, получаем, что существуют такие функция $z \in Z$ и числа $t_{0} \leqslant a_{0} \leqslant b_{0} \leqslant c_{0}$, что $\pi(z)=\left[t_{0}, c_{0}\right], \operatorname{Gr} z \subseteq M,\left(t_{0}, z\left(t_{0}\right)\right) \in S,\left(a_{0}, z\left(a_{0}\right)\right) \in \partial M,\left(c_{0}, z\left(c_{0}\right)\right) \in \partial M$ и $\left(b_{0}, z\left(b_{0}\right)\right) \in H$. Но так как $\left(Z_{M}\right)^{+} S \cap F_{0}=\varnothing, \partial M=F_{0} \cup F_{1}$, то $\left(a_{0}, z\left(a_{0}\right)\right) \in F_{1}$, $\left(c_{0}, z\left(c_{0}\right)\right) \in F_{1}$, т.е. не вьполнено условие $(*)$ для пространства $Z$. Противоречие с условием теоремы.

Итак, существует пространство $Z_{l} \in R_{\text {сеu }}(U)$, для которого выполнены условия, но не вьполнено утверж дение теоремы. Это противоречит доказанному в п. I. Тем самым, теорема доказана.

3. Существование решений. Пусть $U$ - открытое подмножество $\mathbb{R} \times \mathbb{C}^{n}$, пространство $Z_{0} \in R_{c e u}(U)$ является пространством решений линейного дифференциального уравнения $y^{\prime}=A y, y \in \mathbb{C}^{n}$, с постоянной блочно-диагональной матрицей

$$
A=\left(\begin{array}{lll}
A_{1} & & 0 \\
& A_{2} & \\
0 & & A_{3}
\end{array}\right),
$$

причем $\mathbb{C}^{n}=\mathbb{C}^{n_{1}} \oplus \mathbb{C}^{n_{2}} \oplus \mathbb{C}^{n_{3}}, A=A_{1} \oplus A_{2} \oplus A_{3}, A_{i}$ - матрица оператора в $\mathbb{C}^{n_{i}}$; вешественные части собственных значений матрицы $A_{2}$ равны $\mu_{2}$, матрищы $A_{1}$ не меньше $\mu_{1}$, матрицы $A_{3}$ не больше $\mu_{3}$ и $\mu_{1}>\mu_{2}>\mu_{3}$.

Заметим, что это общая ситуация для линейных уравнений с постоянными коэффициентами (после замены базиса в $\mathbb{C}^{n}$ ).

В дальнейшем предполагаем, что для всех $p \in \mathbb{C}^{n_{1}}, q \in \mathbb{C}^{n_{2}}, r \in \mathbb{C}^{n_{3}}$ вьполняются неравенства

$$
\begin{gathered}
\left(\mu_{1}-\varepsilon\right)\|p\|^{2} \leqslant \operatorname{Re}\left(p, A_{1} p\right), \quad\left(\mu_{2}-\varepsilon\right)\|q\|^{2} \leqslant \operatorname{Re}\left(q, A_{2} q\right) \leqslant\left(\mu_{2}+\varepsilon\right)\|q\|^{2} \\
\operatorname{Re}\left(r, A_{3} r\right) \leqslant\left(\mu_{3}+\varepsilon\right)\|r\|^{2}
\end{gathered}
$$

для некоторого $\varepsilon$ такого, что $\mu_{3}+\varepsilon<\mu_{2}-\varepsilon<\mu_{2}<\mu_{2}+\varepsilon<\mu_{1}-\varepsilon$. (Через $(\cdot, \cdot)$ обозначается стандартное скалярное произведение в соответствующих пространствах.)

Выполнения неравенств (1) всегда можно добиться заменой базиса (переходом к псевдожордановой форме, отличающейся от жордановой только тем, что на побочной диагонали стоят не единицы, а $\varepsilon$ ).

Пусть $\theta_{r}>0, \theta_{q}>0$.

Лемма 4. В предположсениях начала п. 3 и (1) имеем:

1. $\left(\|r\|^{2}-\theta_{r}^{2}\|q\|^{2}\right)^{\prime}<0$ в точках, где $\|r\|=\theta_{r}\|q\|,\|q\| \neq 0$;

2. $\left(\|p\|^{2}-\theta_{p}^{2}\|q\|^{2}\right)^{\prime}>0$ в точках, где $\|p\|=\theta_{p}\|q\|,\|q\| \neq 0$

(производная берется в силу системы $\left.y^{\prime}=A y\right)$.

ДоКАЗАТЕЛЬСТВо состоит из простых выкладок:

$$
\begin{aligned}
\left(\|r\|^{2}-\theta_{r}^{2}\|q\|^{2}\right)^{\prime} & =2\left(\operatorname{Re}\left(r, r^{\prime}\right)-\theta_{r}^{2} \operatorname{Re}\left(q, q^{\prime}\right)\right)=2\left(\operatorname{Re}\left(r, A_{3} r\right)-\theta_{r}^{2} \operatorname{Re}\left(q, A_{2} q\right)\right) \\
& \leqslant 2\left(\left(\mu_{3}+\varepsilon\right)\|r\|^{2}-\theta_{r}^{2}\left(\mu_{2}-\varepsilon\right)\|q\|^{2}\right)=2\left(\mu_{3}-\left(\mu_{2}-2 \varepsilon\right)\right) \theta_{r}^{2}\|q\|^{2}<0
\end{aligned}
$$


Аналогично,

$$
\begin{aligned}
\left(\|p\|^{2}-\theta_{p}^{2}\|q\|^{2}\right)^{\prime} & =2\left(\operatorname{Re}\left(p, p^{\prime}\right)-\theta_{p}^{2} \operatorname{Re}\left(q, q^{\prime}\right)\right)=2\left(\operatorname{Re}\left(p, A_{1} p\right)-\theta_{p}^{2} \operatorname{Re}\left(q, A_{2} q\right)\right) \\
& \geqslant 2\left(\left(\mu_{1}-\varepsilon\right)\|p\|^{2}-\theta_{p}^{2}\left(\mu_{2}+\varepsilon\right)\|q\|^{2}\right)=2\left(\mu_{1}-\left(\mu_{2}+2 \varepsilon\right)\right) \theta_{p}^{2}\|q\|^{2}>0
\end{aligned}
$$

Лемма доказана.

СлЕДСТВИЕ 2. За исключением точек $y=(p, q, r)$ с $q=\overline{0}$ граница области в $\mathbb{C}^{n}$, задаваемой неравенством $\|p\|<\theta_{p}\|q\|$, состоит из точек строгого выхода по отношению $к$ системе $y^{\prime}=A y$ (определение см. в $[1, \S \mathrm{X.2}]$ ), а граница области в $\mathbb{C}^{n}$, задаваемой неравенством $\|r\|<\theta_{r}\|q\|,-$ из точек строгого входа.

Teopema 2. Пусmb $\mathbb{C}^{n}=\mathbb{C}^{n_{1}} \oplus \mathbb{C}^{n_{2}} \oplus \mathbb{C}^{n_{3}}$,

$$
A=\left(\begin{array}{lll}
A_{1} & & 0 \\
& A_{2} & \\
0 & & A_{3}
\end{array}\right),
$$

вещественные части собственных значений матрицы $A_{2}\left(n_{2} \times n_{2}\right)$ равны $\mu_{2}$, матрииь $A_{1}\left(n_{1} \times n_{1}\right)$ не меньше $\mu_{1}$, матрицы $A_{3}\left(n_{3} \times n_{3}\right)$ не больше $\mu_{3}\left(\mu_{1}>\mu_{2}>\mu_{3}\right)$. Положим $U=(a, \infty) \times \mathbb{C}^{n}, t^{*}>a, V=U \backslash(\mathbb{R} \times\{\overline{0}\}), \theta_{p}, \theta_{r}, \varepsilon_{r}>0$.

Предположим, что пространство $Z \in\left[R_{\text {сеи }}(U)\right]$ таково, что для всяких последовательностей чисел $\lambda_{i}>0, h_{i} \rightarrow \infty(i \rightarrow \infty)$ пространства $\left(\widetilde{\Phi}_{h_{i} \lambda_{i}}(Z)\right)_{V}$ сходятся $\kappa\left(Z_{0}\right)_{V}$ в $V$, где $\Phi_{h \lambda}$ - замена переменны иию $z(t) \in Z$ в функиию $y(t) \equiv \lambda^{-1} z(t+h)$.

Тогда существует такое $\tau_{1} \geqslant t^{*}$, что для любих $t_{0} \geqslant \tau_{1}, q_{0} \in \mathbb{C}^{n_{2}} \backslash\{\overline{0}\}, r_{0} \in \mathbb{C}^{n_{3}}$, $\left\|r_{0}\right\| \leqslant \theta_{r}\left\|q_{0}\right\|$, найдутся $p_{0} \in \mathbb{C}^{n_{1}} \quad$ и функиия $z(t)=(p(t), q(t), r(t)) \in Z^{+}$maкuе, что $\pi(z)=\left[t_{0}, \infty\right), p\left(t_{0}\right)=p_{0}, q\left(t_{0}\right)=q_{0}, r\left(t_{0}\right)=r_{0} u\|p(t)\| \leqslant \theta_{p}\|q(t)\|$, $\|r(t)\| \leqslant\left(\theta_{r}+\varepsilon_{r}\right)\|q(t)\|$ для всех $t \geqslant t_{0}$.

ДокАЗАТЕЛЬСТво. Перейдем в координаты, в которых вьполнено (1). Пусть $0<$ $\varepsilon_{p}<\theta_{p}$. Введем обозначения

$$
\begin{aligned}
& G_{p}=\left\{(t, y)=(t, p, q, r):(t, y) \in V,\|p\|<\theta_{p}\|q\|\right\}, \\
& H_{p}=\left\{(t, y)=(t, p, q, r):(t, y) \in V,\|p\|<\left(\theta_{p}-\varepsilon_{p}\right)\|q\|\right\}, \\
& G_{r}=\left\{(t, y)=(t, p, q, r):(t, y) \in V,\|r\|<\left(\theta_{r}+\varepsilon_{r}\right)\|q\|\right\}, \\
& H_{r}=\left\{(t, y)=(t, p, q, r):(t, y) \in V,\|r\|<\theta_{r}\|q\|\right\} .
\end{aligned}
$$

I. Покажем, что существует такое $\tau_{2} \geqslant t^{*}$, что для всех $z \in Z_{V}^{+}$, удовлетворяющих условиям $\tau_{2} \leqslant t=\inf \pi(z),(t, z(t)) \in \partial_{V} G_{p}$, вьполнено $\operatorname{Gr} z \cap H_{p}=\varnothing$.

Предположим противное. Тогда существует такая последовательность функций $z_{k} \in$ $Z_{V}$ с областями определения $\left[t_{k}, s_{k}\right]$, что $\left(t_{k}, z_{k}\left(t_{k}\right)\right) \in \partial_{V} G_{p},\left(s_{k}, z_{k}\left(s_{k}\right)\right) \in H_{p}, t_{k} \rightarrow \infty$ при $k \rightarrow \infty$. Ограничивая области определения функций $z_{k}$, можно считать, что $\operatorname{Gr} z_{k} \subseteq$ $\left[G_{p}\right]_{V}$.

Рассмотрим замены переменных $\Phi_{h_{k} \lambda_{k}}$ с $h_{k}=t_{k}-t^{*}$ и $\lambda_{k}=\left\|z_{k}\left(t_{k}\right)\right\|$. Введем обозначения $T=\left\{s_{k}-t_{k}: k \in \mathbb{N}\right\}, y_{k}=\widetilde{\Phi}_{h_{k} \lambda_{k}}\left(z_{k}\right)(k \in \mathbb{N})$. Возможны два случая. 
а) Последовательность $T$ ограничена. Тогда в силу сходимости пространств $\widetilde{\Phi}_{h_{k} \lambda_{k}}(Z)_{V}$ к $\left(Z_{0}\right)_{V}$ в $V$ существует такая функция $y_{0} \in\left(Z_{0}\right)_{V}$, что некоторая подпоследовательность последовательности $y_{k}$ сходится к $y_{0}$. Пусть $\pi\left(y_{0}\right)=\left[t^{*}, s_{0}\right]$. Тогда $\left(t^{*}, y_{0}\left(t^{*}\right)\right) \in \partial_{V} G_{p},\left(s_{0}, y_{0}\left(s_{0}\right)\right) \in\left[H_{p}\right]$. Получили противоречие со следствием 2 .

б) Последовательность $T$ не ограничена. Пусть $\left\{y_{k_{l}}: l \in \mathbb{N}\right\}$ - подпоследовательность последовательности $\left\{y_{k}: k \in \mathbb{N}\right\}$, для которой вьполнено $s_{k_{l}}-t_{k_{l}} \rightarrow \infty$ при $l \rightarrow \infty$. Обозначим через $z_{l}^{*}$ продолжение функции $y_{k_{l}}$ до функции из $\widetilde{\Phi}_{h_{k_{l}} \lambda_{k_{l}}}(Z)_{V}^{+}$. По теореме IX.3.12 из [3] в силу сходимости пространств $\widetilde{\Phi}_{h_{k} \lambda_{k}}(Z)_{V} \mathrm{k}\left(Z_{0}\right)_{V}$ в $V$ существует такая функция $z_{0} \in\left(Z_{0}\right)_{V}^{+}$, что некоторая подпоследовательность последовательности функций $z_{l}^{*}$ сходится к ней на любом отрезке в ее области определения. Отсюда $t^{*}=\inf \pi\left(z_{0}\right),\left(t^{*}, z_{0}\left(t^{*}\right)\right) \in \partial_{V} G_{p}$ и $\operatorname{Gr} z_{0} \subseteq\left[G_{p}\right]_{V}$. Это противоречит следствию 2.

II. Покажем теперь, что существует такое $\tau_{3} \geqslant t^{*}$, что для всех $z \in Z_{V}^{+}$, удовлетворяющих условиям $\tau_{3} \leqslant t=\inf \pi(z),(t, z(t)) \in H_{r}$, выполнено $\operatorname{Gr} z \subseteq G_{r}$.

Предположим противное. Аналогично п. І получаем последовательность таких функций $z_{k}$ из $Z_{V}$, что $\pi\left(z_{k}\right)=\left[t_{k}, s_{k}\right],\left(t_{k}, z_{k}\left(t_{k}\right)\right) \in H_{r},\left(s_{k}, z_{k}\left(s_{k}\right)\right) \in \partial_{V} G_{r}, t_{k} \rightarrow \infty$ $(k \rightarrow \infty)$. Без ограничения общности можем считать, что Gr $z_{k} \subseteq\left[G_{r}\right]_{V} \backslash \operatorname{Int} H_{r}$.

Рассмотрим замены переменных $\Phi_{h_{k} \lambda_{k}} \mathrm{c} h_{k}=t_{k}-t^{*}$ и $\lambda_{k}=\left\|z_{k}\left(t_{k}\right)\right\|$. Тогда последовательность $\widetilde{\Phi}_{h_{k} \lambda_{k}}(Z)_{V}$ сходится к $\left(Z_{0}\right)_{V}$ в $V$.

Рассуждая как в п. I, можно доказать, что либо

а) существует такая функция $y_{0} \in\left(Z_{0}\right)_{V}$, что $\pi\left(y_{0}\right)=\left[t^{*}, s\right],\left(t^{*}, y_{0}\left(t^{*}\right)\right) \in H_{r}$, $\left(s, y_{0}(s)\right) \in \partial_{V} G$, либо

б) существует такая функция $z_{0} \in\left(\left(Z_{0}\right)_{V}\right)^{+}$, что inf $\pi\left(z_{0}\right)=t^{*},\left(t^{*}, z_{0}\left(t^{*}\right)\right) \in H_{r}$ и $\operatorname{Gr} z_{0} \subseteq\left[G_{r}\right]_{V} \backslash \operatorname{Int} H_{r}$.

Но и то, и другое противоречит следствию 2.

III. Положим $M=\left[G_{p}\right]_{V} \cap\left[G_{r}\right]_{V}, F_{0}=\partial_{V} M \cap \partial_{V} G_{r}, F_{1}=\partial_{V} M \cap \partial_{V} G_{p}$. Пусть, кроме того, $H=M \cap\left[H_{p}\right]_{V}, S=\left\{\left(t_{0}, p, q_{0}, r_{0}\right) \in V: p \in \mathbb{C}^{n_{1}},\|p\| \leqslant \theta_{p}\left\|q_{0}\right\|\right\}$ для произвольных $t_{0} \geqslant \max \left\{\tau_{3}, \tau_{2}\right\}, q_{0} \in \mathbb{C}^{n_{2}} \backslash\{\overline{0}\}, r_{0} \in \mathbb{C}^{n_{3}}$ таких, что $\left\|r_{0}\right\| \leqslant \theta_{r}\left\|q_{0}\right\|$. Нетрудно видеть, что $S \subseteq H_{r}$. Отсюда и из п. II следует, что $\left(Z_{M}\right)^{+} S \cap F_{0}=\varnothing$. Из п. I следует, что выполнено условие $(*)$.

Для введенных объектов вьполнены все предположения теоремы 1. Согласно этой теореме существует такая функция $z \in Z^{+}, z(t)=(p(t), q(t), r(t))$, что inf $\pi(z)=t_{0}$, $q\left(t_{0}\right)=q_{0}, r\left(t_{0}\right)=r_{0}$ и $\|p(t)\| \leqslant \theta_{p}\|q(t)\|,\|r(t)\| \leqslant\left(\theta_{r}+\varepsilon_{r}\right)\|q(t)\|, t \in \pi(z)$. Пусть $u_{0}=\left(t_{0}, q_{0}, r_{0}\right)$. Обозначим полученную функцию через $z_{u_{0}}$.

IV. Доказательство леммы будет завершено, если мы покажем, что сушествует такое $\tau_{1} \geqslant \max \left\{\tau_{3}, \tau_{2}\right\}$, что все функции $z_{u_{0}} \in Z^{+}$, построенные в п. III при $u_{0}=\left(t_{0}, q_{0}, r_{0}\right)$, $t_{0} \geqslant \tau_{1}$, имеют областью определения $\left[t_{0}, \infty\right)$. Предположим противное. Тогда существуют такие $u_{k}=\left(t_{k}, q_{k}, r_{k}\right)$, что $t_{k} \rightarrow \infty$ при $k \rightarrow \infty, \pi\left(z_{u_{k}}\right)=\left[t_{k}, T_{k}\right), T_{k}<\infty$. Зафиксируем произвольное $b>0$. Пусть $s_{k}=t_{k}, z_{k}=z_{u_{k}}$, если $T_{k}-t_{k} \leqslant b$, и $s_{k}=T_{k}-b$, $z_{k}=\left.z_{u_{k}}\right|_{\left[s_{k}, T_{k}\right)}$ в противном случае.

Согласно теореме IX.3.12 из [3], примененной к последовательности функций $\widetilde{\Phi}_{h_{k} \lambda_{k}}\left(z_{k}\right)$, где $h_{k}=s_{k}-t^{*}, \lambda_{k}=\left\|z_{k}\left(s_{k}\right)\right\|$, существует такая функция $z_{0} \in\left(Z_{0}^{+}\right)_{V}$, $z_{0}(t)=\left(p_{0}(t), q_{0}(t), r_{0}(t)\right)$, что $\left\|p_{0}(t)\right\| \leqslant \theta_{p}\left\|q_{0}(t)\right\|,\left\|r_{0}(t)\right\| \leqslant\left(\theta_{r}+\varepsilon_{r}\right)\left\|q_{0}(t)\right\|$. Кроме того, для всех $t \in \pi\left(z_{0}\right)$ и для всякого отрезка $I \subseteq \pi\left(z_{0}\right)$, начиная с некоторого $k$, функции $\left.\widetilde{\Phi}_{h_{k} \lambda_{k}}\left(z_{k}\right)\right|_{I}$ определены и сходятся к $\left.z_{0}\right|_{I}$. Отсюда следует, что $\pi\left(z_{0}\right)=\left[t^{*}, T_{0}\right)$, где $T_{0}-t^{*} \leqslant b$, т.е. $\sup \pi(z)<\infty$. 
Но, как известно, для пространства решений $Z_{0}$ системы уравнений $y^{\prime}=A y$ такое невозможно. Доказательство завершено.

Вешественный случай мало чем отличается от уже рассмотренного комплексного.

TЕорема 3. Пусть $\mathbb{R}^{n}=\mathbb{R}^{n_{1}} \oplus \mathbb{R}^{n_{2}} \oplus \mathbb{R}^{n_{3}}$,

$$
A=\left(\begin{array}{lll}
A_{1} & & 0 \\
& A_{2} & \\
0 & & A_{3}
\end{array}\right),
$$

вещественные части собственных значений матрицы $A_{2}\left(n_{2} \times n_{2}\right)$ равны $\mu_{2}$, матрицы $A_{1}\left(n_{1} \times n_{1}\right)$ не меньше $\mu_{1}$, матрицы $A_{3}\left(n_{3} \times n_{3}\right)$ не больше $\mu_{3}\left(\mu_{1}>\mu_{2}>\mu_{3}\right)$.

Полохим $U=(a, \infty) \times \mathbb{R}^{n}, t^{*}>a, V=U \backslash(\mathbb{R} \times\{\overline{0}\}), \theta_{p}, \theta_{r}, \varepsilon_{r}>0$.

Предполохим, что $Z \in\left[R_{\text {сеи }}(U)\right]$ таково, что для всяких последовательностей $\lambda_{i}>0, h_{i} \rightarrow \infty(i \rightarrow \infty)$ пространства $\left(\widetilde{\Phi}_{h_{i} \lambda_{i}}(Z)\right)_{V}$ сходятся $\kappa\left(Z_{0}\right)_{V}$ в $V$, где $Z_{0}$ - пространство решений дифференииального уравнения $y^{\prime}=A y$.

Тогда существует такое $\tau_{1} \geqslant t^{*}$, что для любы $t_{0} \geqslant \tau_{1}, q_{0} \in \mathbb{R}^{n_{2}} \backslash\{\overline{0}\}, r_{0} \in \mathbb{R}^{n_{3}}$, $\left\|r_{0}\right\| \leqslant \theta_{r}\left\|q_{0}\right\|$, найдутся $p_{0} \in \mathbb{R}^{n_{1}}$ и функиия $z(t)=(p(t), q(t), r(t))$ из $Z^{+}$maкuе, что $\pi(z)=\left[t_{0},+\infty\right), p\left(t_{0}\right)=p_{0}, q\left(t_{0}\right)=q_{0}, r\left(t_{0}\right)=r_{0} u\|p(t)\| \leqslant \theta_{p}\|q(t)\|$, $\|r(t)\| \leqslant\left(\theta_{r}+\varepsilon_{r}\right)\|q(t)\|$ для всех $t \geqslant t_{0}$.

ДокАЗАТЕЛЬСТво. Введем в качестве скалярного произведения вещественную часть стандартного эрмитового произведения в псевдожордановом базисе матрицы $A$. Тогда для всех $p \in \mathbb{R}^{n_{1}}, q \in \mathbb{R}^{n_{2}}, r \in \mathbb{R}^{n_{3}}$ выполняются неравенства

$$
\begin{gathered}
\left(\mu_{1}-\varepsilon\right)\|p\|^{2} \leqslant\left(p, A_{1} p\right), \quad\left(\mu_{2}-\varepsilon\right)\|q\|^{2} \leqslant\left(q, A_{2} q\right) \leqslant\left(\mu_{2}+\varepsilon\right)\|q\|^{2} \\
\left(r, A_{3} r\right) \leqslant\left(\mu_{3}+\varepsilon\right)\|r\|^{2}
\end{gathered}
$$

для некоторого $\varepsilon$ такого, что $\mu_{3}+\varepsilon<\mu_{2}-\varepsilon<\mu_{2}<\mu_{2}+\varepsilon<\mu_{1}-\varepsilon$.

Последующая часть доказательства вполне аналогична доказательству теоремы 2 .

4. Пример. Пусть $U=\mathbb{R} \times\left(\mathbb{R}^{3} \backslash\{\overline{0}\}\right)$. В качестве примера применения теоремы 3 рассмотрим систему

$$
\left\{\begin{array}{l}
p^{\prime}=2 p \\
q^{\prime}=q+q^{2} /(t(|r-p|+|r-q|)), \\
r^{\prime}=-r
\end{array}\right.
$$

где $p, q, r \in \mathbb{R}$, а производная берется по переменной $t \in \mathbb{R}$. Заметим, что под решениями мы понимаем абсолютно непрерьвные функции, производные которых почти всюду удовлетворяют уравнениям рассматриваемой системы. Соответствующее пространство решений в области $U$ обозначим $Z$. Для любого $N \in \mathbb{N}$ пусть $Z_{N}$ - пространство решений системы дифференциальных уравнений

$$
\left\{\begin{array}{l}
p^{\prime}=2 p \\
q^{\prime}=f_{N}(t, p, q, r) \\
r^{\prime}=-r
\end{array}\right.
$$


где $f_{N}(t, p, q, r)=q+q^{2} /(t(|r-p|+|r-q|))$ на множестве

$$
\left\{(t, p, q, r) \in U: q+\frac{q^{2}}{t(|r-p|+|r-q|)}<N\right\}
$$

и $f_{N}(t, p, q, r)=N$ в остальных точках множества $U$. Нетрудно видеть, что $Z_{N} \in R_{c e u}(U)$ и последовательность пространств $\left\{Z_{N}: N \in \mathbb{N}\right\}$ сходится к пространству $Z$ в $U$, т.е. $Z \in\left[R_{\text {сеи }}(U)\right]$.

Введем следующие обозначения: $S=\{(t, p, q, r) \in U: p=q=r\}, V=U \backslash S$. Пусть, кроме того, $Z_{i}=\widetilde{\Phi}_{h_{i} \lambda_{i}}(Z), i \in \mathbb{N}$, где $\lambda_{i}>0, h_{i} \rightarrow \infty$ при $i \rightarrow \infty$. Пусть $Z_{0} \in R_{\text {ceu }}(U)-$ пространство решений системы дифференциальных уравнений

$$
\left\{\begin{array}{l}
p^{\prime}=2 p \\
q^{\prime}=q \\
r^{\prime}=-r
\end{array}\right.
$$

Из [3, теорема IX.4.9] следует, что последовательность пространств $\left(Z_{i}\right)_{V}$ сходится в $V$ к пространству $\left(Z_{0}\right)_{V}$.

Покажем, что последовательность пространств $Z_{i}$ равностепенно непрерьвна, т.е. $\left\{Z_{i}: i \in \mathbb{N}\right\} \in s(U)$. Предположим противное. Пусть $K$ компактное подмножество $U$ и последовательность функций $z_{n} \in\left(Z_{i_{n}}\right)_{K}\left(i_{1}<i_{2}<\cdots\right)$ не является равностепенно непрерьвной. Пусть тогда число $\varepsilon>0$ и точки $a_{k}<b_{k}$ из области определения $z_{n_{k}}$ таковы, что $\left|a_{k}-b_{k}\right|<1 / k,\left\|z_{n_{k}}\left(a_{k}\right)-z_{n_{k}}\left(b_{k}\right)\right\| \geqslant \varepsilon$. Пусть $z_{k}^{*}=\left.z_{n_{k}}\right|_{\left[a_{k}, b_{k}\right]}$. В силу компактности метризуемого пространства $\exp K$ (см. теоремы IV.5.1 и IV.7.3 в [3]) можно предположить, что последовательность $\mathrm{Gr} z_{k}^{*}$ сходится в топологии Виеториса к некоторому множеству $F \in \exp K$ (в противном случае перейдем к подпоследовательности, удовлетворяющей этому свойству). Из теоремы IV.8.8 в [3] следует, что множество $F$ связно. Из условия $\left|a_{k}-b_{k}\right|<1 / k$ и теоремы IV.8.6 в [3] следует, что $\left[a_{k}, b_{k}\right]$ сходятся к некоторому одноточечному множеству $\{t\}$, в которое отображается множество $F$ при проектировании на первьй сомножитель произведения $\mathbb{R} \times \mathbb{R}^{3}$. Из выбора числа $\varepsilon$ следует, что диаметр множества $F$ не меньше $\varepsilon$.

Докажем, что $F \cap V \neq \varnothing$. Пусть, напротив, $F \subseteq S$. Пусть отображение П - проектирование в $\mathbb{R} \times \mathbb{R}^{3}$ на все переменные, кроме $q$. Имеем: графики всех функций из $Z_{i}$ (для всех $i \in \mathbb{N} \cup\{0\})$ проектируются посредством П на графики решений системы дифференциальных уравнений

$$
\left\{\begin{array}{l}
p^{\prime}=2 p \\
r^{\prime}=-r
\end{array}\right.
$$

Так как пространство решений этой системы лежит в $R_{c e}\left(\mathbb{R} \times\left(\mathbb{R}^{2} \backslash\{\overline{0}\}\right)\right)($ см. $[2$, теорема 2.1]), найдется такое решение $\zeta$ этой системы, что для некоторой подпоследовательности $z_{k_{l}}^{*}$ последовательности функций $z_{k}^{*}$ последовательность множеств $\Pi\left(\operatorname{Gr} z_{k_{l}}^{*}\right)$ сходится к $\operatorname{Gr} \zeta$. С другой стороны, $\Pi\left(\operatorname{Gr} z_{k}^{*}\right) \rightarrow \Pi(F)$. Так как $\pi(\zeta)=\{t\}$, множество $\operatorname{Gr} \zeta=\Pi(F)$ одноточечное. Но $\left.\Pi\right|_{S}$ - инъективное отображение и если $F \subseteq S$, то $F$ одноточечное множество. Мы получили противоречие с тем, что диаметр $F$ не меньше $\varepsilon$.

Итак, существует такая точка $A \in \mathbb{R}^{3}$, что $(t, A) \in F \cap V$. Тогда можно выбрать такое компактное подмножество $K_{0}$ области $V$, что $(t, A) \in \operatorname{Int} K_{0}, F \cap \partial K_{0} \neq \varnothing$ (иначе, беря убывающую последовательность таких компактных множеств и используя связность $F$, получим опять, что $F$ одноточечное множество). Из теоремы IV.3.10 в [3] следует, что, начиная с некоторого $k$, мы можем так выбрать отрезки $I_{k} \subseteq \pi\left(z_{k}^{*}\right)$ с концами $s_{k}$ и $t_{k}$, что $\left(s_{k}, z_{n_{k}}\left(s_{k}\right)\right) \rightarrow(t, A),\left(t_{k}, z_{n_{k}}\left(t_{k}\right)\right) \in \partial K_{0},\left.\operatorname{Gr} z_{n_{k}}\right|_{I_{k}} \subseteq K_{0}$. Графики 
элементов последовательности $\alpha=\left\{\left.z_{n_{k}}\right|_{I_{k}}: k \in \mathbb{N}\right\}$ лежат в компакте $K_{0} \subseteq V$, и так как $\left\{\left(Z_{i}\right)_{V}: i \in \mathbb{N}\right\} \in s(V)$ (это следует из отмеченной ранее сходимости последовательности $\left(Z_{i}\right)_{V}$ в $\left.V\right)$, из последовательности $\alpha$ можно выбрать сходящуюся подпоследовательность. Но предел этой подпоследовательности должен быть функцией, определенной на одноточечном множестве $\{t\}$, график которой пересекается с $\partial K$ и Int $K$. Это невозможно. Полученное противоречие доказывает, что $\left\{Z_{i}: i \in \mathbb{N}\right\} \in s(U)$.

Покажем теперь, что последовательность пространств $Z_{i}$ сходится к $Z_{0}$ в $U$. Определим функцию $f$ на $\mathbb{R} \times \mathbb{R}^{3}$ равенством $f(t, z)=p-r$, где $t \in \mathbb{R}, z=(p, q, r) \in \mathbb{R}^{3}$. По предложению IX.4.15 в [3] для всякого $c>0$ множество

$$
\begin{aligned}
M(f, c)= & \left\{z \in C_{s}(U): f(t, z(t))-f(s, z(s)) \geqslant c(t-s)\right. \\
& \text { для всех } s, t \in \pi(z), s<t\},
\end{aligned}
$$

а также аналогично определяемое множество $M(-f, c)$ замкнуты в $C_{s}(U)$. По предложению IX.4.16 в [3] множество $S$ не более чем счетно относительно пространств $M(f, c)$ и $M(-f, c)$. Для $c \geqslant 0$ введем обозначения

$$
\begin{aligned}
W_{c}^{+} & =\{(t, p, q, r) \in U: r+2 p>c\}, \\
W_{c}^{-} & =\{(t, p, q, r) \in U: r+2 p<-c\}, \\
W_{c} & =W_{c}^{+} \cup W_{c}^{-} .
\end{aligned}
$$

Так как для функции $z(t)=(p(t), q(t), r(t))$ изпространства $Z_{i}$ вьполнено $p^{\prime}-r^{\prime}=r+2 p$, то при $c>0$ имеем $\left(Z_{i}\right)_{W_{c}^{+}} \subseteq M(f, c)$ и $\left(Z_{i}\right)_{W_{c}^{-}} \subseteq M(-f, c)$. Таким образом, по теореме IX.4.9 из [3], для всякого $c>0$ последовательности пространств $\left(Z_{i}\right)_{W_{c}^{+}}$и $\left(Z_{i}\right)_{W_{c}^{-}}$сходятся к пространствам $\left(Z_{0}\right)_{W_{c}^{+}}$и $\left(Z_{0}\right)_{W_{c}^{-}}$в $W_{c}^{+}$и $W_{c}^{-}$соответственно. Так как $W_{0}=\cup\left\{W_{c}: c>0\right\}$ и множества $W_{c}$ открыты и упорядочены по включению, то любой компакт $K \subseteq W_{0}$ лежит в $W_{c}$ для некоторого $c>0$. Поэтому последовательность пространств $\left(Z_{i}\right)_{W_{0}}$ сходится к пространству $\left(Z_{0}\right)_{W_{0}}$ в $W_{0}$. Если $p=r \neq 0$, то $r+2 p=3 r \neq 0$. Следовательно, $S \subseteq W_{0}$, а значит, $W_{0} \cup V=U$. В силу теоремы IX.4.9 в [3] отсюда следует, что последовательность пространств $Z_{i}$ сходится к пространству $Z_{0}$ в $U$.

Таким образом, для нашего примера вьполнена теорема 3.

Приведенньй пример не покрьвается классическими результатами об асимптотическом интегрировании квазилинейных дифференциальных уравнений (см. [1, глава Х]). Действительно, правая часть нашей системы не допускает локальной мажоранты класca $L_{1}$.

\section{СПИСОК ЦИТИРОВАННОЙ ЛИТЕРАТУРЫ}

[1] Хартман $\Phi$. Обыкновенные дифференциальные уравнения. М.: Мир, 1970.

[2] Филиппов В.В. Топологическое строение пространств решений обыкновенных диффференциальных уравнений // УМН. 1993. № 1. С. 103-154.

[3] Федорчук В. В., Филиппов В. В. Общая топология. Основные конструкции. М.: Изд-во МГУ, 1988.

[4] Michael E. Continuous selections I // Ann. Math. 1956. V. 63. P. 361-382.

(С. Р. Габдрахманов, В.В. Филиппов) Московский государственный университет (Б. С. Клебанов) Московский институт повьштения квалификации работников образования E-mail : gabdr@nw.math.msu.su 